shortages at a time of growing population, more challenging healthcare delivery and increased societal expectation. This has created a highly challenging psychosocial environment for healthcare workers. Consultants feel undervalued and are concerned about the quality of care they provide. They perceive care to be thwarted by managers being reactive and not focused on longterm planning. As well as feeling undervalued, trainees too have concerns about the quality of care they provide and they struggle to manage both service and training demands.

Method Utilising validated questionnaires, a national cross-sectional survey of hospital doctors, undertaken in 2014, sought responses from consultants and trainees working in the sector. The response rate was 55\%.

Results Hospital doctors in Ireland had higher levels of psychological distress than elsewhere. They also had significant symptoms of depression and anxiety as well as high levels of burnout and occupational stress. Self-stigma in relation to mental illness was more common in doctors than in the general population. However, current desire to practice remained high.

Discussion The high levels of personal and workplace distress identified in this study suggest that much needs to be done to highlight the importance of doctors' wellbeing in this country. Self-stigmatisation is likely a barrier to early identification and treatment of mental health problems. Post-graduate training bodies have already begun to address these issues with trainee and trainer members. Occupational health services have a key role to play in ensuring appropriate access to care and in determining necessary workplace restrictions and/or supports for this group.

\section{OCCUPATIONAL HEALTH AND EPIDEMIOLOGICAL METHODS RESEARCH: CHALLENGES AND OPPORTUNITIES}

Jain Rajiv Kumar. Vice President, Association of Environmental and Occupational HealthDelhi

\subsection{6/oemed-2018-ICOHabstracts.20}

Purpose It is widely accepted that there is definite need for strengthening research in developing, validating and refining epidemiological methods for application in Occupational Health, if preventive strategies have to be devised on actual evidence. There is need to further identify and manage the challenges and also diffuse the opportunities available for collaboration in conducting research in Epidemiological Methods for Occupational Health.

Review and discussion Developing improved methods for exposure assessment, statistical analysis, studying migrant workers and other vulnerable populations, the use of biomarkers, and new hazards provide challenges and opportunities.

Issues of challenges due to lack of validated statistical tools, complexity of ethical considerations, lack of training in occupational epidemiological methods, non-clarity in methods for accurate determination of proportion of disease/diseases attributable to occupation, shifting of hazardous industries from developed to developing countries, lack of collaboration mechanisms with veterinary epidemiologists, lack of participatory research, effective and appropriate communicating tools, preventability paradox and maintaining public health perspective to ensure proper population perspective, shall be discussed.
Conclusion and outcome Opportunities for developing, nurturing, financing and implementing local, regional and global collaboration through fair, transparent, democratic and participative consortiums/forums/alliances of people, civil society and public and private institutions and Governments, in the field of epidemiological methods research shall be explored and discussed. A case for germinating an Institute of Occupational Health Metrics and Evaluation in a developing country environment shall be built upon.

\section{DEVELOPMENT OF OCCUPATIONAL HEALTH NURSING ASSESSMENT TOOLS FOR INDIVIDUAL AND GROUP/ ORGANISATION}

Keiko Kono. Research Centre for Occupational Health Nursing, Yokkaichi Nursing and Medical Care University/Japan

\subsection{6/oemed-2018-ICOHabstracts.21}

Introduction In order to provide high quality activities in occupational health $(\mathrm{OH})$ nursing, it is essential to assess the worker and the group/organisation. However, these kind of tools have not been available until we developed the $\mathrm{OH}$ nursing assessment tools. Methods we have developed the assessment tools for both individual and group/organisation by the task force consisting of $17 \mathrm{OH}$ nurses from 2000 through 2014. In the development of the tool for Individual, we based on the frame work of North American Nursing Diagnosis International (NANDA I), and for group/organisation we referred to the Community as Partner Model by Anderson and the Hanasaari Conceptual Model which was developed in the 1 st International Symposium on $\mathrm{OH}$ Nursing Education in Finland in 1988.

Results The tool for Individual has been divided into 13 domains with a face sheet. By using the face sheet, we can capture work/life style history of individual effectively. The 13 domains consist of Health Promotion, Nutrition, Elimination, Activity/Rest, Perception/Cognition, Self-Perception, Role Relationships, Sexuality, Coping/Stress Tolerance, Life Principle, Safety/Protection, Comfort and Growth/Development. The tool for group/organisation has been comprised 8 core items (Company Outline, Organisation Outline, Constitution of the Worker, Human Resources/Labour Management/Education, Culture, Labour Condition, Health Status and Safety/Health), which directly related with the worker belonging to the group/organisation, and 5 sub-items (Administration, Economy, Environment, Social Resource and Traffic), which will be indirectly related with the worker.

Conclusion These tools are useful to catch the information about workers and their surroundings including labour standpoint. As the result, currently many $\mathrm{OH}$ nurses in Japan utilise these tools in daily work that can help them to assess the worker holistically and to assess the group/organisation comprehensively and systematically.

\section{KNEE ARTHROPLASTY AND THE UNFORESEEN IMPACT ON WORK}

Paul FM Kuijer. Academic Medical Centre, University of Amsterdam, Coronel Institute of Occupational Health, People and Work Outpatient Clinic, Amsterdam Public Health research institute, Amsterdam, the Netherlands

10.1136/oemed-2018-ICOHabstracts.22 